\title{
Sunitinib - vom Paradigmenwechsel zu einem Therapiestandard beim metastasierten Nierenzellkarzinom (mRCC)
}

\author{
Lothar Bergmann \\ Medizinische Klinik II, Hämatologie/Onkologie, J.W. Goethe-Universität, Frankfurt/M.
}

\author{
Schlüsselwörter \\ Metastasiertes Nierenzellkarzinom · Erstlinientherapie . \\ Zielgerichtete Therapie - Small molecules . \\ Tyrosinkinase-Hemmer · Sunitinib
}

\section{Zusammenfassung}

Zielgerichtete Substanzen haben in der Behandlung des metastasierten Nierenzellkarzinoms (mRCC) zu einem Paradigmenwechsel geführt. Der Multi-Kinase-Inhibitor Sunitinib konnte sich innerhalb kurzer Zeit als ein neuer Standard in der Erstlinientherapie etablieren. In einem umfassenden Studienprogramm wurde Sunitinib ausgehend von Phase-II-Studien in der Zweitlinientherapie über eine Phase-III-Studie zur Erstlinientherapie bis hin zum breiten Einsatz in der klinischen Praxis systematisch entwickelt. Die Prognose für Patienten mit mRCC hat sich durch die Einführung des Multi-Kinase-Hemmers deutlich verbessert: Gegenüber Interferon-alpha wurde mit Sunitinib eine Verdopplung des progressionsfreien Überlebens und eine Vervierfachung der Ansprechrate erreicht. Die hohe Wirksamkeit übersetzte sich auch in das mediane Gesamtüberleben von über 2 Jahren im Sunitinib-Arm der Studie. Zusammen mit den konsistenten Ergebnissen des internationalen Sunitinib-Expanded-Access-Programms verfügt die Substanz über eine breite Evidenzbasis und umfassende praktische Erfahrung für den Einsatz in allen Patientenpopulationen. Dies spiegelt sich auch in den Empfehlungen nationaler und internationaler Fachgesellschaften wider. In Deutschland ist Sunitinib die am häufigsten eingesetzte Substanz beim mRCC.

\section{Zielgerichtete Therapie beim mRCC}

Mit der Identifizierung biologischer Zielstrukturen hat sich in der Krebstherapie insgesamt eine grundlegende Wende vollzogen. Auch beim metastasierten Nierenzellkarzinom (mRCC) führte die darauf basierende Entwicklung von zielgerichteten Medikamenten zu einem Paradigmenwechsel. Eine Erkrankung, die bis vor wenigen Jahren noch als weitgehend therapierefraktär galt, wurde behandel-
Key Words

Metastatic renal cell carcinoma - First-line therapy .

Targeted therapy . Small molecules . Tyrosine kinase inhibitors . Sunitinib

\section{Summary}

Sunitinib - from Paradigm Shift to Standard Treatment of Metastatic Renal Cell Carcinoma

Targeted therapies have led to a paradigm shift in the treatment of metastatic renal cell carcinoma (mRCC). The multi-kinase inhibitor Sunitinib was rapidly established as a new standard in first-line therapy. Sunitinib was systematically developed in a comprehensive study program starting with phase II studies in second-line therapy, progressing to a phase III study of first-line therapy and ending with broad application in clinical practice. The prognosis for patients with mRCC has significantly improved with the introduction of the multi-kinase inhibitor: Sunitinib has doubled progression-free survival and quadrupled the response rate compared to Interferon alpha. This benefit is supported by more than 2 years overall survival in the Sunitinib arm of the study. In combination with the consistent results of the international Sunitinib Expanded Access Program, the substance possesses a broad evidence base on extensive practical application in all patient populations. This is also reflected in the recommendations of national and international professional associations. Sunitinib is the most frequently applied drug for mRCC in Germany.

bar, und die Prognose von Nierenkrebspatienten hat sich deutlich verbessert. Nicht zuletzt hat diese Entwicklung auch das Therapieziel in dieser Situation entscheidend verändert: Im Mittelpunkt steht nicht mehr nur Palliation, sondern Lebensverlängerung bei erhaltener Lebensqualität. Maßgeblichen Anteil an dieser Therapieverbesserung hat der orale Multi-Kinase-Inhibitor Sunitinib (Sutent ${ }^{\circledR}$ ), das erste zielgerichtete Medikament, das zur First-line-Therapie des mRCC zugelassen wurde.

\section{KARGER}

Fax +497614520714

Information@Karger.de

www.karger.com (c) 2010 S. Karger GmbH, Freiburg

www.karger.com/onk
Prof. Dr. med. Lothar Bergmann

Tumorzentrum Rhein-Main, Medizinische Klinik II

Zentrum der Inneren Medizin

Klinikum der J.W. Goethe-Universität Frankfurt/M

Theodor-Stern-Kai 7

60590 Frankfurt 


\section{Sunitinib-Studienprogramm beim mRCC}

Abb. 1. Studienprogramm zur Entwicklung von Sunitinib beim metastasierten Nierenzellkarzinom.

\begin{tabular}{|c|c|c|c|c|c|}
\hline Studie & $\mathrm{n}$ & Phase & $\begin{array}{l}\text { Therapie- } \\
\text { regime }\end{array}$ & $\begin{array}{l}\text { Therapie- } \\
\text { linie }\end{array}$ & Ergebnisse \\
\hline $\begin{array}{l}\text { Rosenberg } \\
\text { George } \\
\text { (gepoolte } \\
\text { Analyse) }\end{array}$ & 169 & II & $\begin{array}{l}\text { Sunitinib } \\
\text { (einarmig) }\end{array}$ & Zweitlinie & $\begin{array}{l}\text { PFS median: } 8,3 \\
\text { Ansprechrate: } 49 \% \\
\text { OS median: } 20 \\
\text { Monate }\end{array}$ \\
\hline $\begin{array}{l}\text { Motzer } 5 \\
\text { Figlin }\end{array}$ & 750 & III & $\begin{array}{l}\text { Sunitinib } \\
\text { vs. IFN-a }\end{array}$ & Erstlinie & $\begin{array}{l}\text { PFS median: } 5 \text { vs. } \\
11 \text { Monate }(p<0,001) \\
\text { OS median: } 26,4 \\
\text { Monate (Sunitinib); } \\
21,8 \text { Monate (IFN-a) } \\
(p=0,051)\end{array}$ \\
\hline Gore $^{7}$ & 4.564 & $\begin{array}{l}\text { Expanded- } \\
\text { Access- } \\
\text { Programm }\end{array}$ & Sunitinib & $\begin{array}{l}76 \% \text { vor- } \\
\text { therapiert }\end{array}$ & $\begin{array}{l}\text { PFS median (gesamt): } \\
\text { 10,9 Monate } \\
\text { Klinischer Nutzen } \\
\text { (CR+PR-SD): } 76 \%\end{array}$ \\
\hline
\end{tabular}

Noch vor wenigen Jahren unbefriedigende Therapiesituation

Der enorme Fortschritt durch die Einführung von TyrosinkinaseHemmern wie Sunitinib wird besonders deutlich, wenn man sich die Therapiesituation noch vor 4 Jahren vergegenwärtigt: Onkologische Standardverfahren wie Chemo- und Strahlentherapie sind beim mRCC weitgehend wirkungslos; die mittlere Überlebenszeit der Patienten lag bei 9-12 Monaten. Einen gewissen Fortschritt brachte die Immuntherapie mit Zytokinen wie Interferon-alpha (IFN- $\alpha$ ) und/ oder Interleukin-2 (IL-2), die teilweise in Kombination mit Chemotherapeutika angewendet wurde. Allerdings konnte auch damit nur eine 5-Jahres-Überlebenswahrscheinlichkeit von $10 \%$ erreicht werden; das mediane Überleben betrug zwischen 12 und 15 Monaten [1]. Eine Cochrane-Metaanalyse, die 53 zwischen 1966 und 2003 publizierte Studien auswertete, ermittelte für die Zytokintherapie ein medianes Überleben von 13,3 Monaten; der mittlere Überlebensvorteil gegenüber den Vergleichsarmen betrug lediglich 3,8 Monate [2].

\section{Second-line: Effektive Therapie nach Zytokinversagen}

Sunitinib war beim mRCC eine der ersten Substanzen mit zielgerichtetem Wirkmechanismus, deren Wirksamkeit sich in der klinischen Erprobung bestätigte. So evaluierten zwei unabhängige Phase-IIStudien Sunitinib auch nach Versagen einer Therapie mit Zytokinen als hocheffektive Therapieoption.

In einer gepoolten Analyse der zwei einarmigen multizentrischen Studien mit 169 Patienten wurde eine objektive Ansprechrate von $45 \%$ und erstmals ein progressionsfreies Überleben (PFS) von 8,4 Monaten erreicht. Die mediane Dauer des Ansprechens betrug 11,9 Monate, das mediane Gesamtüberleben (OS) 19,9 Monate. Nach 30 Monaten lebten noch $40 \%$ der Patienten. Zudem war eine Komplettremission zu verzeichnen. $32 \%$ der Patienten hatten eine stabile Erkrankung ( $\mathrm{SD} \geq 3$ Monate).

Diese Daten führten im Juli 2006 zu einer Zulassung für die Zweitlinientherapie [3, 4].
First-line: Verdopplung des PFS gegenüber IFN- $\alpha$

Eine randomisierte Phase-III-Studie mit 750 Patienten erbrachte auch in der Erstlinientherapie überzeugende Ergebnisse [5], die im Januar 2007 in eine Zulassungserweiterung mündeten. Beim primären Endpunkt, dem PFS, erreichten die Patienten im Sunitinib-Arm im Vergleich zu IFN- $\alpha$ eine Verdopplung (median 11 vs. 5 Monate; $\mathrm{p}<0,001)$. Die objektive Ansprechrate war mit 47 versus $12 \%$ gegenüber IFN- $\alpha$ vervierfacht $(p<0,001)$. Ein auf dem ASCO 2008 vorgestelltes Update ergab für die ITT-Population ein Gesamtüberleben von 26,4 Monaten unter Sunitinib; in der Gruppe mit IFN- $\alpha$ waren es 21,8 Monate $(\mathrm{p}=0,051)$ [6].

Aktuelle Daten aus einem erweiterten Studienprogramm stützen die positiven Ergebnisse aus den klinischen Studien und bestätigen die gute Wirksamkeit von Sunitinib in der breiten Anwendung [7]. So liefert das breit angelegte Sunitinib-Expanded-Access-Programm mit mehr als 4500 Patienten konsistente Daten zur Effektivität und Verträglichkeit von Sunitinib, insbesondere auch bei schwierig zu behandelnden Subgruppen.

\section{Bevorzugte Therapie der ersten Wahl}

Unter den derzeit beim mRCC verfügbaren Substanzen weist Sunitinib eine breite und valide Evidenz in verschiedenen Therapiesituationen auf (Abb. 1). Inzwischen steht der Wirkstoff in mehr als 76 Ländern zur Verfügung. Über zwei Drittel der First-line-mRCC-Patienten in Europa erhalten Sunitinib; in Deutschland ist Sunitinib mit Abstand die am häufigsten eingesetzte Substanz in dieser Indikation [8-11]. Sunitinib hat die Immuntherapie als einstigen Standard abgelöst und kann heute als ein neuer Standard in der Therapie des mRCC bezeichnet werden. Aufgrund der breiten Evidenzbasis wird Sunitinib von den meisten Experten bei Patienten mit niedrigem oder intermediärem Risiko als Therapie der ersten Wahl eingesetzt [12]. In den folgenden Beiträgen werden die Daten, die den Stellenwert von Sunitinib in der Therapie des mRCC belegen, näher erläutert. 


\section{Disclosure Statement}

The author is a member of the advisory boards for Novartis, Roche, Pfizer, and Wyeth and participates in clinical trials in RCC funded by Novartis, Pfizer, and Wyeth.

\section{Literatur}

1 Motzer RJ, Bacik J, Murphy BA, Russo P, Mazumdar M: Interferon-alfa as a comparative treatment for clinical trials of new therapies against advanced renal cell carcinoma. J Clin Oncol 2002;20:289-296.

2 Coppin C, Porzsolt F, Awa A, Kumpf J, Coldman A, Wilt T: Immunotherapy for advanced renal cel cancer. Cochrane Database Syst Rev 2005;(1): CD001425.

3 Rosenberg JE, Michaelson MD, Redman BG, et al: Sunitinib therapy for patients with metastatic renal cell carcinoma: updated results of two phase II trials and prognostic factor analysis for survival ASCO 2007, poster 5095

4 George DJ, Michaelson MD, Rosenberg JE, et al: Sunitinib in patients with cytokine-refractory metastatic renal cell carcinoma (mRCC). Eur J Cancer 2007;5:304s, abstr 4517.
5 Motzer RJ, Hutson TE, Pharm D, et al: Sunitinib versus interferon alfa in metastatic renal-cell carcinoma. N Engl J Med 2007;356:115-124.

6 Figlin RA, Hutson TE, Tomczak P, et al: Overall survival with sunitinib versus interferon (IFN) alfa as first-line treatment of metastatic renal cell carcinoma (mRCC). J Clin Oncol 2008;26(suppl):abstr 5024.

7 Gore ME, Szczylik C, Porta C, et al: Safety and efficacy of sunitinib for metastatic renal-cell carcinoma: an expanded-access trial. Lancet Oncol 2009;10:757-763.

8 Müller L, Münch A, Blumenstengel K, et al: Treatment of patients with advanced or metastatic renal cell carcinoma (RCC registry): a 'real life' treatment overview of non-trial patients in outpatient cancer centres in Germany. DGO 2009, abstr V861.
9 Ljungberg B, Hanbury DC, Kuczyk MG, et al: Guidelines on renal cell carcinoma. Update 2009. www.uroweb.org/professional-resources/guidelines/online.

10 National Comprehensive Cancer Network Practice Guidelines in Oncology: Kidney Cancer v2.2010.

11 Miller K, Bergmann L, Albers P, et al: Interdisziplinäre Empfehlungen zur Behandlung des metastasierten Nierenzellkarzinoms. Akt Urol 2009;40: 27-30.

12 Interdisziplinäres Expertengespräch Nierenzellkarzinom der Deutschen Krebsgesellschaft 2009. www. krebsgesellschaft.de/expertengespraech_rcc_2009. 